\title{
Large artery ischemic stroke in severe COVID-19
}

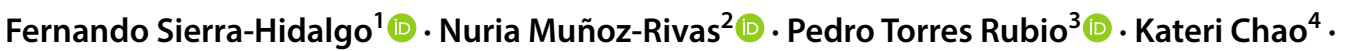 \\ Mercedes Villanova Martínez ${ }^{5} \cdot$ Paz Arranz García $^{6}$ - Eva Martínez-Acebes ${ }^{1}$
}

Received: 14 May 2020 / Revised: 28 May 2020 / Accepted: 30 May 2020 / Published online: 27 June 2020

(c) Springer-Verlag GmbH Germany, part of Springer Nature 2020

\section{Dear Sirs,}

The coronavirus disease 2019 (COVID-19) pandemic has spread rapidly all around the world. Between March 5th and April 12th, 2020, more than 1200 patients with a positive reverse transcriptase PCR for SARS-CoV-2 were admitted to our secondary hospital. Eight of them had concurrent ischemic stroke during the viral disease. Here, we describe the clinical and imaging features, and outcome, of these patients.

Stroke occurred, while in hospital in six patients and at home in the other two patients. All of them met the diagnostic criteria for severe COVID-19 as previously defined [1]. Detailed description of cases is provided in the Supplementary Material (Online Resources 1,2). One was a woman and seven $(88 \%)$ were men, with a median age of 68.5 years (Table 1). None of them had had a previous stroke. Hypertension was the most common vascular risk factor $(63 \%)$. Four patients were on antithrombotic therapy

Electronic supplementary material The online version of this article (https://doi.org/10.1007/s00415-020-09967-1) contains supplementary material, which is available to authorized users.

Fernando Sierra-Hidalgo

fernando.sierragh@salud.madrid.org

1 Department of Neurology, Hospital Universitario Infanta Leonor, Avenida Gran Vía del Este 80, 28031 Madrid, Spain

2 Department of Internal Medicine, Hospital Universitario Infanta Leonor, Madrid, Spain

3 Department of Radiology, Unidad Central de Radiodiagnóstico, Hospital Universitario Infanta Leonor, Madrid, Spain

4 Department of Anesthesiology, Critical Care, and Pain Management, Hospital Universitario Infanta Leonor, Madrid, Spain

5 Critical Care Unit, Hospital Universitario Infanta Leonor, Madrid, Spain

6 Department of Medical Administration, Hospital Universitario Infanta Leonor, Madrid, Spain prior to admission: three patients were on antiplatelets (one as a secondary prevention after myocardial infarction, and two as a primary prevention due to a high vascular risk); and one on acenocoumarol due to atrial fibrillation. All the patients who suffered in-hospital strokes were on standard thromboprophylaxis with enoxaparine since admission except for the patient with atrial fibrillation, who was on subcutaneous enoxaparine $60 \mathrm{mg}$ bid. Ischemic stroke occurred a median of 11.5 days after the onset of COVID-19 symptoms (interquartile range, IQR 2.8-16.3). Among hospitalized patients, stroke occurred a median of 5.5 days after admission (IQR 3.5-7.5). Bilateral lung infiltrates on chest $\mathrm{X}$-ray were present in all. At the time of stroke, turbidimetric $\mathrm{D}$ dimer was $>2,000 \mu \mathrm{g} / \mathrm{l}$ in 63\% (5/8) of patients (median 2,515 $\mu \mathrm{g} / \mathrm{l}$; IQR 985-15,500; normal value $<500 \mu \mathrm{g} / \mathrm{l}$ ). Antiphospholipid antibodies were not obtained.

Overall, five strokes involved one cerebral arterial territory and three involved two or more arterial territories. All of them were large artery infarctions as diagnosed by clinical and cranial CT findings (four anterior circulation infarctions, three posterior circulation infarctions, and one with both anterior and posterior circulation infarctions). Magnetic resonance imaging was not performed on any patient. Only one patient met definite TOAST criteria for the diagnosis of large artery atherosclerotic infarction, and another one had a probably cardioembolic stroke due to preexisting atrial fibrillation (incomplete evaluation) [2]. None of the other six patients met diagnostic criteria for atherosclerotic, cardioembolic, or small vessel ischemic stroke (three with cryptogenic strokes, and three with incomplete evaluation). Intraarterial thrombi with absence of significant atherosclerotic plaques were observed in the intracranial or supra-aortic arteries in three out of four patients in which $\mathrm{CT}$ angiograms were obtained. Four patients did not undergo $\mathrm{CT}$ angiography due to a worsening in their respiratory and neurological performance despite therapy. Limitation of the therapeutic effort was applied in these cases, and patients died early after the stroke diagnosis without additional diagnostic workup. Two patients had other thrombotic disorders 
Table 1 Characteristics of eight patients with COVID-19 and ischemic stroke
Demographics

Female gender, no. (\%)

1 (12)

Age (years), median (IQR)

$68.5(59-77.3)$

$0(0-2.5)$

Hypertension, no. (\%)

$5(63)$

Diabetes, no. (\%)

3 (38)

Dyslipidemia, no. (\%)

4 (50)

BMI $\left(\mathrm{kg} / \mathrm{m}^{2}\right)$, median (IQR)

Smoking, no. (\%)

$27.2(24.9-29.1)$

$0(0)$

$0(0)$

1 (13)

1 (13)

Atrial fibrillation, no. (\%)

Antiplatelet, no. (\%)

Treatments

Oxygen therapy

Oxygen supplementation, no. (\%)

$4(50)$

NIMV, no. (\%)

1 (13)

IMV, no. (\%)

3 (38)

ICU admission, no. (\%)

$3(38)$

Hydroxychloroquine, no. (\%)

7 (88)

Azithromycin, no. (\%)

7 (88)

Corticoids, no. (\%)

$6(75)$

Tocilizumab, no. (\%)

$5(63)$

Previous thromboprophylaxis, no. (\%)

$6(75)$

Stroke presentation

Time from COVID-19 onset to stroke (days), median (IQR)

$11.5(2.8-16.3)$

Time from admission to stroke (days), median (IQR)

$5.5(3.5-7.5)$

Stroke place

At home, no. (\%)

$2(25)$

$6(75)$

In-hospital, no. (\%)

$27(15.8-39.3)$

NIHSS score, median (IQR)

$2,515(985-15,500)$

No. of cerebral arterial territories involved

1, no. $(\%)$

5 (63)

$>1$, no. $(\%)$

3 (37)

Arterial territories involved

ACA, no. (\%)

$0(0)$

MCA, no. (\%)

$5(63)$

PCA, no. (\%)

3 (37)

$3(37)$

$3(75)$

Thrombus on CT angiogram $(n=4)$, no. $(\%)$

Outcome (mRS)

$\leq 2$, no. $(\%)$

$3-5$, no. $(\%)$

6, no. $(\%)$

$4(50)$

$A C A$ anterior cerebral artery, $B A$ basilar artery, $B M I$ body mass index, $I C U$ intensive care unit, $I Q R$ interquartile range, $I V M$ invasive mechanical ventilation, $M C A$ middle cerebral artery, $m R S$ modified Rankin scale, NIHSS National Institute of Health stroke scale, NIVM non-invasive mechanical ventilation, PCA posterior cerebral artery 
(one with a pulmonary embolism, patient no. 8 in Supplementary table; and another one with a floating aortic arch thrombus, patient no. 3).

None of the patients met criteria for receiving reperfusion therapies of the occluded arteries. Two of them were started on acetylsalicylic acid, and four received subcutaneous enoxaparine $1 \mathrm{mg} / \mathrm{kg}$ bid. On evolution, four patients $(50 \%)$ died, one remains in a minimally conscious state, one has a severe focal neurological deficit (left middle cerebral artery syndrome), and two have moderate focal neurological deficits, after a median follow-up of 29 days for survivors.

In this series of eight patients, although the evidence is limited by its observational nature and sample size, severe COVID-19 was associated with non-atherosclerotic, large artery ischemic strokes. A high frequency of vertebrobasilar territory involvement was noted, and most patients did not meet diagnostic criteria for common causes of ischemic stroke [3]. Observed cumulative incidence of ischemic stroke during the period included in this series largely exceeds the expected incidence for our 1,200 admitted subjects during the 39 days evaluated [4].

At this point, in the growing knowledge about the mechanisms underlying the high morbidity and mortality associated to COVID-19, an atypical and enhanced form of acute coagulopathy secondary to endothelial disfunction and an inflammation-mediated prothrombotic state seem to be playing an important role. In the context of severe disease, vascular wall inflammation can initiate thrombus formation by activating endothelial cells, platelets, and leukocytes, which would trigger the coagulation pathway [5-7]. This may induce a prothrombotic state that confers a high risk for ischemic stroke, either by a direct damage to a previously healthy endothelial wall or by enhancing a thrombotic effect in cases where a specific cause, such as atherosclerosis, is present $[8,9]$. If larger prospective studies confirm these observations, hypercoagulability associated with COVID19 might be a contributory cause for large vessel ischemic stroke. Until robust evidence is available, the observation of intraarterial thrombi in the absence of significant atherosclerosis among these patients warrants consideration of individualized enhanced thromboprophylaxis for hospitalized patients with severe forms of SARS-CoV-2 infection.

Acknowledgments To the members of the "Infanta Leonor Thrombosis Research Group"; to Dr. Pablo Ryan; and to Darryl Solochek for editing the manuscript.
Funding None.

\section{Compliance with ethical standard}

Conflicts of interest On behalf of all authors, the corresponding author states that there is no conflict of interest.

Ethical standard This study belongs to the COVID-19@Vallecas cohort. The study has been approved by the Hospital Universitario Infanta Leonor ethics committee and has, therefore, been performed in accordance with the ethical standards laid down in the 1964 Declaration of Helsinki and its later amendments, and with the Spanish data protection laws.

Consent to participate Written informed consent was waived due to the retrospective nature of the study. Collected data were anonymized, and each patient was identified by a unique alphanumeric identification code.

\section{References}

1. Guan WJ, Ni ZY, Hu Y et al (2020) Clinical Characteristics of coronavirus disease 2019 in China. N Engl J Med 382:1708-1720

2. Adams HP Jr, Bendixen BH, Kappelle LJ et al (1993) Classification of subtype of acute ischemic stroke. Definitions for use in a multicenter clinical trial. TOAST. Trial of Org 10172 in acute stroke treatment. Stroke 24:35-41

3. Yaghi S, Ishida K, Torres J et al (2020) SARS2-CoV-2 and stroke in a New York Healthcare System. Stroke STROKEAHA120030335. doi: https://doi.org/10.1161/STROK EAHA.120.030335

4. Díaz-Guzmán J, Egido JA, Gabriel-Sánchez R et al (2012) Stroke and transient ischemic attack incidence rate in Spain: the IBERICTUS study. Cerebrovasc Dis 34:272-281

5. Han H, Yang L, Liu R et al (2020) Prominent changes in blood coagulation of patients with SARS-CoV-2 infection. Clin Chem Lab Med 2020 (pii: /j/cclm.ahead-of-print/cclm-2020-0188/cclm2020-0188.xml). doi: https://doi.org/10.1515/cclm-2020-0188

6. Varga Z, Flammer AJ, Steiger P et al (2020) Endothelial cell infection and endotheliitis in COVID-19. Lancet 395:1417-1418. https ://doi.org/10.1016/S0140-6736(20)30937-5

7. Beyrouti R, Adams ME, Benjamin L et al (2020) Characteristics of ischaemic stroke associated with COVID-19. J Neurol Neurosurg Psychiatry (pii: jnnp-2020-323586) doi: https://doi. org/10.1136/jnnp-2020-323586

8. Merad M, Martin JC (2020) Pathological inflammation in patients with COVID-19: a key role for monocytes and macrophages. Nat Rev Immunol. https://doi.org/10.1038/s41577-020-0331-4

9. Oxley TJ, Mocco J, Majidi S et al (2020) Large-vessel stroke as a presenting feature of Covid-19 in the young. $\mathrm{N}$ Engl $\mathrm{J}$ Med 382:e60 